\title{
The Spontaneous Nature of Lightning Initiation Revealed
}

\author{
C. Sterpka ${ }^{1 *}$, J. Dwyer ${ }^{1}$, N. Liu ${ }^{1}$, B.M. Hare ${ }^{2}$, O. Scholten ${ }^{2-4}$, \\ S. Buitink ${ }^{5,6}$, S. ter Veen $^{7}$, A. Nelles ${ }^{8,9}$ \\ ${ }^{1}$ Space Science Center (EOS), Department of Physics and Astronomy, University of New Hampshire, \\ Durham NH, USA \\ ${ }^{2}$ University Groningen, Kapteyn Astronomical Institute, Landleven 12, 9747 AD Groningen, The \\ Netherlands \\ ${ }^{3}$ University of Groningen, KVI Center for Advanced Radiation Technology, Groningen, The Netherlands \\ ${ }^{4}$ Interuniversity Institute for High-Energy, Vrije Universiteit Brussel, Pleinlaan 2, 1050 Brussels, Belgium \\ ${ }^{5}$ Department of Astrophysics/IMAPP, Radboud University Nijmegen, Nijmegen, The Netherlands \\ ${ }^{6}$ Astrophysical Institute, Vrije Universiteit Brussel, Pleinlaan 2, 1050 Brussels, Belgium \\ ${ }^{7}$ Netherlands Institute for Radio Astronomy (ASTRON), Dwingeloo, The Netherlands \\ ${ }^{8}$ Erlangen Center for Astroparticle Physics, Friedrich-Alexander-Univeristät Erlangen-Nürnberg, Germany \\ ${ }^{9}$ DESY, Platanenallee 6, 15738 Zeuthen, Germany
}

\section{Key Points:}

- As seen in VHF, the first lightning signal detectable above background increases exponentially by two orders of magnitude in $15 \mu \mathrm{s}$.

- Initiation is likely caused by branching streamers with overall constant propagation speed of $4.8 \pm 0.1 \times 10^{6} \mathrm{~m} / \mathrm{s}$ during the exponential ramp-up phase.

- Mechanism is similar to narrow-bipolar events, but much weaker in VHF power. 


\begin{abstract}
Here, we present new radio interferometer beamforming observations of lightning initiation using data from the Low Frequency Array (LOFAR). We show that the first lightning source in the flash increases exponentially in intensity by two orders of magnitude in 15 microseconds, while propagating 88 meters away from the initiation location at a constant speed of $4.8 \pm 0.1 \times 10^{6} \mathrm{~m} / \mathrm{s}$. A second source replaces the first source at the initiation location, and subsequent propagation of the lightning leader follows. We interpret the first source to be a rapidly propagating and intensifying positive streamer discharge that subsequently produces a hot leader channel near the initiation point. How lightning initiates is one of the greatest unsolved problems in the atmospheric sciences, and these results shed light on this longstanding mystery.
\end{abstract}

\title{
Plain Language Summary
}

\section{Introduction}

The basic principle of radio interferometry is that radio signals measured by separate antennas from a single source add coherently when adjusted for propagation time delays, while pulses from different sources or from random noise add incoherently (Taylor et al., 1999). For a lightning source, the combined signals will result in a received power approximately proportional to the square of the number of antennas and inversely proportional to the square of the distance from each antenna to the source. In contrast, signals from random noise will result in received power approximately proportional to the number of antennas. LOFAR is comprised of thousands of VHF antennas that are distributed all over Europe. For lightning studies, antennas are selected from the Netherlands to provide both large and small antenna separations (also known as baselines). The combination of the low-noise antennas and long baselines provides outstanding image resolution due to the fact that the maximum achievable angular resolution is proportional to $\lambda / d$, where $\lambda$ is the wavelength of the radiation and $d$ is the largest baseline length. Interferometers previously used to study lightning typically consisted of 3-4 antennas separated by a few hundred meters resulting in a resolutions on the order of $1.6^{\circ}$ azimuth and $3.5^{\circ}$ in elevation with no sensitivity along the radial axis (Tilles et al., 2019). In many cases, the algorithm used is closer to a time-of-arrival technique where only the location of the peaks are extracted from the result of the cross-correlations (Rison et al., 2016; Stock et al., 2014). The LOFAR impulsive imager uses a similar technique to the timeof-arrival, but has the advantage of hundreds of antennas and large baselines (Scholten, Hare, Dwyer, Sterpka, et al., 2021). As a result, the impulsive imager achieves source densities of over 200 sources per millisecond (Scholten, Hare, Dwyer, Sterpka, et al., 2021). Within this work and the previous impulsive imager, we achieve angular resolutions up to 1 arc second in azimuth and 2 arc seconds in elevation. This results in sub-meter resolution along both the horizontal (azimuth) and elevation axes, while also achieving 5 $\mathrm{m}$ resolution along the radial axis. LOFAR beamforming combines hundreds of antennas and selects baselines of up to 100 kilometers, resulting in images with remarkably high signal to noise ratios and resolutions produced with sensitivity below the noise level of the galactic background (gb) on individual antennas (Hare et al., 2018; Scholten, Hare, Dwyer, Liu, et al., 2021). The gb units are derived from the normalized noise level of the galactic and thermal background and represents the sensitivity limit for a single LOFAR antenna. To find the absolute power the antenna response must be taken into account, and as it has not been included within this study we use the convenient gb units.

\section{Results}

On August 9th, 2018, a thunderstorm developed in Western Europe ((KNMI), 2018). At 14:14 UTC, a lightning flash initiated $29 \mathrm{~km}$ west and $6 \mathrm{~km}$ south from the LOFAR core at an altitude of about $6 \mathrm{~km}$. A large number of impulsive sources were located with 

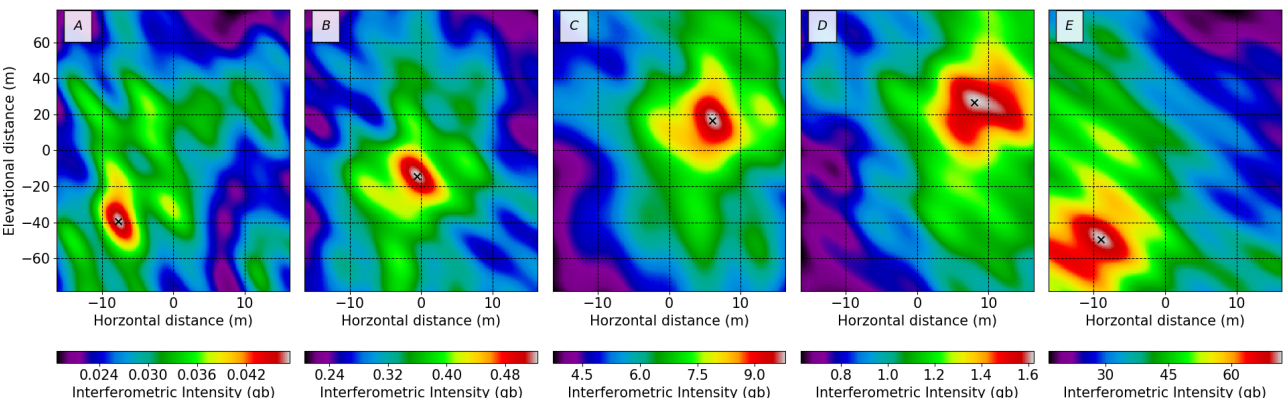

Figure 1. Image of initiation event from 2018 flash. Images are sequential from left (A) to right $(\mathrm{E})$. The color scale at the bottom shows interferometric beamforming intensity (note that the scale is different for each image). The black $\mathrm{x}$ indicates the location of peak intensity for each image. The imaging origin begins and ends at $6.1456 \mathrm{~km}$ South, $28.5129 \mathrm{~km}$ West, and 6.2542 $\mathrm{km}$ in altitude from the LOFAR core. The radial direction extends from the LOFAR core to the image center such that the image plane is perpendicular to the radial axis. The radial distance is adjusted so that each image contains the source maximum. The time of each image is indicated in (Figure 2) by the matching labels and corresponding section of the intensity curve.

LOFAR through the impulsive imager (see supplemental figures S4 and S5), with the first located source at approximately $22 \mu$ s after the low-intensity activity revealed by the beamformed observations (Scholten, Hare, Dwyer, Sterpka, et al., 2021). The impulsive imager is efficient at locating impulsive or short duration high-intensity pulses. However, unlike interferometric beamforming, it is not well suited for identifying features with low intensities or broad time structures, both of which are found to occur during initiation (Marshall et al., 2014, 2019).

Figure 1 shows interferometric images of the initiation of the 2018 lightning flash, created from radiation in the $30-80 \mathrm{MHz}$ portion of the very high frequency (VHF) band on 114 antennas with the longest baseline being $100 \mathrm{~km}$. The intensity peak in the top left panel (labeled A) shows the first detected source, representing the initiation of the flash and has an intensity of about $0.05 \mathrm{gb}$. Panel B shows the source moving rapidly upward to the right while increasing in intensity. The third panel (C) shows the source at peak intensity. In panel $\mathrm{D}$, the source has decreased in intensity while still moving. In panel E, the first source vanishes and is replaced by a new source that forms within $6 \mathrm{~m}$ of the initiation location first seen in panel A. Following this, the first impulsive imager located source develops about $11 \mathrm{~m}$ from the first source seen in panel A and develops into an initial leader in the following millisecond (see supplemental figures S4 and S5).

For the initiation event, all images were generated using pixel sizes of approximately $16 \mathrm{~cm}$ along the horizontal axis, $78 \mathrm{~cm}$ on the vertical axis, and $10 \mathrm{~m}$ along the radial axis. Each image has an integration time of $0.5 \mu$ s for all antennas. Note that the shape of the images are nontrivial and do not necessarily correspond to the shape of the lightning source; it is product of the layout of the antenna beams. The total distance the source traveled from start to end of coherent emission was about $88 \mathrm{~m}$. The distance from the start of the initiation event to the the first impulsive imager located source was approximately $11 \mathrm{~m}$ (or about $99 \mathrm{~m}$ from the end of the coherent emission).

For (Figures 2 and 3), the intensity and location of the brightest pixel in each image integrated over a microsecond was identified. In order to correctly locate the voxel with peak intensity, images are also created parallel to the radial axis (not shown). This procedure ensures that we are implementing true $3 \mathrm{D}$ imaging and improves the accu- 


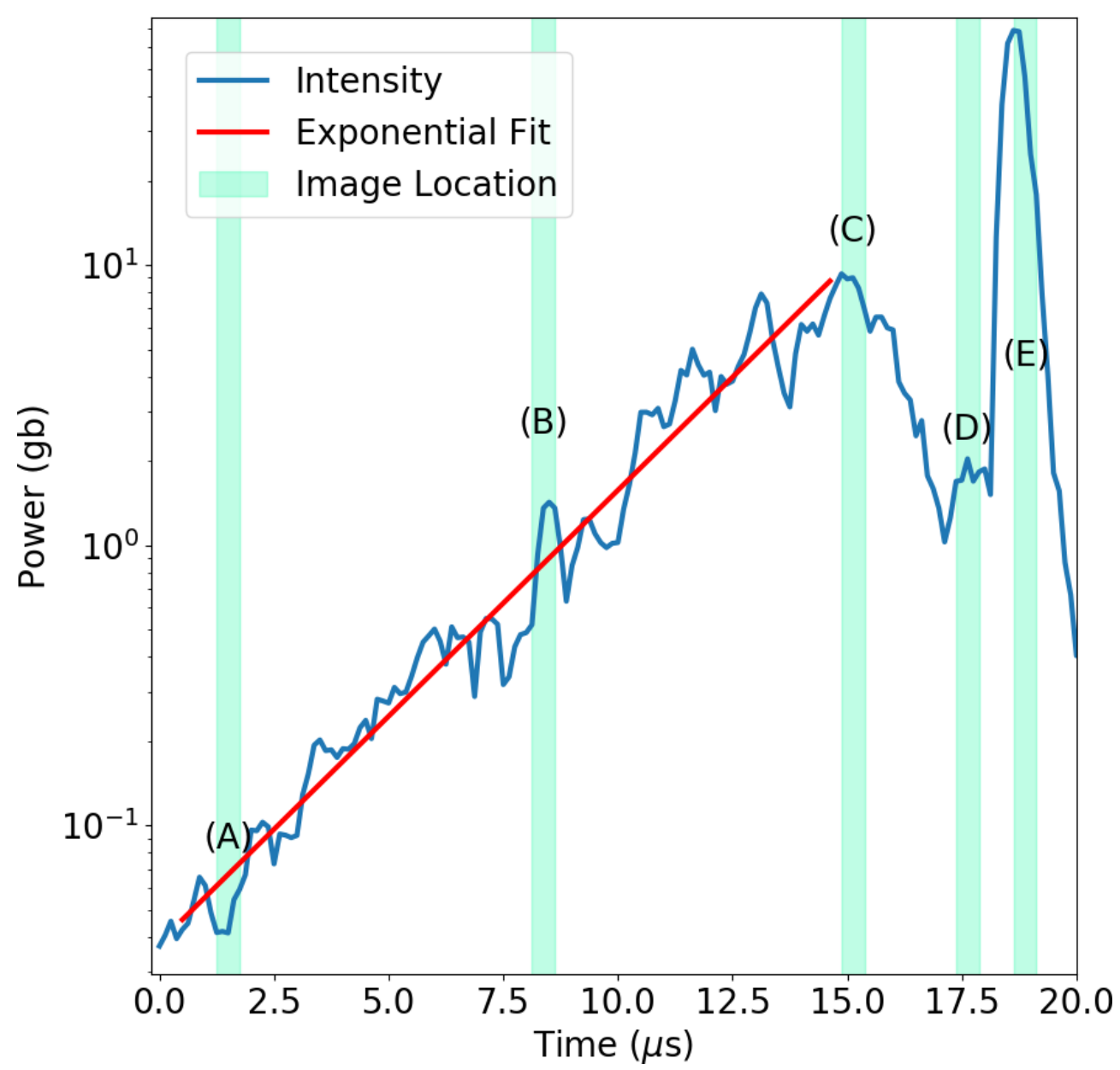

Figure 2. VHF power versus time, showing exponential increase in the power. Plot shows the source ramp-up for VHF emission prior to the first impulsive imager located lightning source for the 2018 flash. The blue curve is the source intensity, the red line is an exponential fit. The green shaded regions identify the sections of the intensity trace that corresponds to the imaging windows used to produce panels (A)-(E) in (Figure 1). 
racy of locating the source in three dimensions. These data were then used to calculate the source ramp-up and velocity as shown in the figures. A fit was then performed on both the position versus time and the source intensity vs time.

The VHF source power displayed in (Figure 2) was averaged over $0.5 \mu$ s and calculated at the location of brightest pixel. The figure demonstrates that the source power increased exponentially over a $15.0 \mu$ s time period with a $2.7 \pm 0.4 \mu$ s e-folding time. The source power then quickly decreased an order of magnitude over $2 \mu$ s while still maintaining a constant velocity. Within the following microsecond, a second source was observed near the location of the initiation point. As the initiation event lasted for more that $15 \mu \mathrm{s}$, it must have been generated by many independent VHF sources. (da Silva \& Pasko, 2013; Shi et al., 2016; Petersen et al., 2008).

The fit in (Figure 3) yielded an overall speed of $4.8 \pm 0.1 \times 10^{6} \mathrm{~m} / \mathrm{s}$. To achieve this, the locations of the sources used in calculating the velocity fit are measured to within a precision of tens of centimeters along the horizontal and vertical axes. The intensities of many of these sources are below the noise level of a single antenna for a lightning event approximately $30 \mathrm{~km}$ from the LOFAR core. The speed of this event paired with the rampup rate results in an e-folding length of $13.0 \pm 1.9 \mathrm{~m}$. What is particularly surprising about (Figures 2 and 3 ) is that the speed is constant over a two order of magnitude increase in intensity followed by an order of magnitude drop in intensity. This suggests an underlying steady-state process, however it is not clear how one would model the observed changes in intensity while also maintaining a constant velocity.

\section{Discussion}

The sources presented were the first detectable activity of the flash. This was confirmed by checking a $1 \mathrm{~km}$ region around the initiation event for a time period of $1 \mathrm{~ms}$ beforehand. Within the time period before the initiation, we identified only a slightly higher than average noise level. However, within $2.5 \mu$ s of the initiation event, there was an even higher maximum background of about $0.25 \mathrm{gb}$ due to interference from a remote flash. There were no sources located in the initiation region of the reported flash at the observed baseline level of $0.25 \mathrm{gb}$ for the data affected by the remote flash and no sources above the mean background rate of $0.01 \mathrm{gb}$ at any other point in the $1 \mathrm{~ms}$ time period.

The initiation event is seen to exponentially increase in power from observed background, followed by propagation away from the initiation point at a velocity on the order of $10^{6} \mathrm{~m} / \mathrm{s}$ for nearly one hundred meters. The power then rapidly decreases followed by the observation of the first impulsive radio source that later develops into the initial leader (Marshall et al., 2019; Stolzenburg et al., 2020). A possible explanation of these observations is a streamer avalanche similar to the model originally developed by Griffiths and Phelps in 1976 (Griffiths \& Phelps, 1976). Streamers are ionizing and self-propagating discharge processes that can take place in virgin air (Dwyer \& Uman, 2014). A streamer can be initiated on a hydrometeor, which is any water or ice particle formed in the atmosphere. Since hydrometeors can become polarized, the electric fields near their surfaces can become enhanced, thereby initiating discharges (Shi et al., 2019; Dubinova et al., 2015). If the ambient thunderstorm electric field is sufficiently high as it propagates, the streamer can branch multiple times, forming an avalanche of streamers while producing VHF radiation (Liu et al., 2012). As the avalanche propagates, it can produce significant charge separation and heating, which then results in the formation of a hot leader channel (Petersen et al., 2008; Phelps, 1974; Attanasio et al., 2019; Luque \& Ebert, 2014).

Figure 4 illustrates this interpretation of the LOFAR observations. Starting with the left panel in (Figure 4) the initiation starts at (a) with a single positive streamer (b). The streamer carries positive charge at its tip and leaves negative charge in its wake. The 

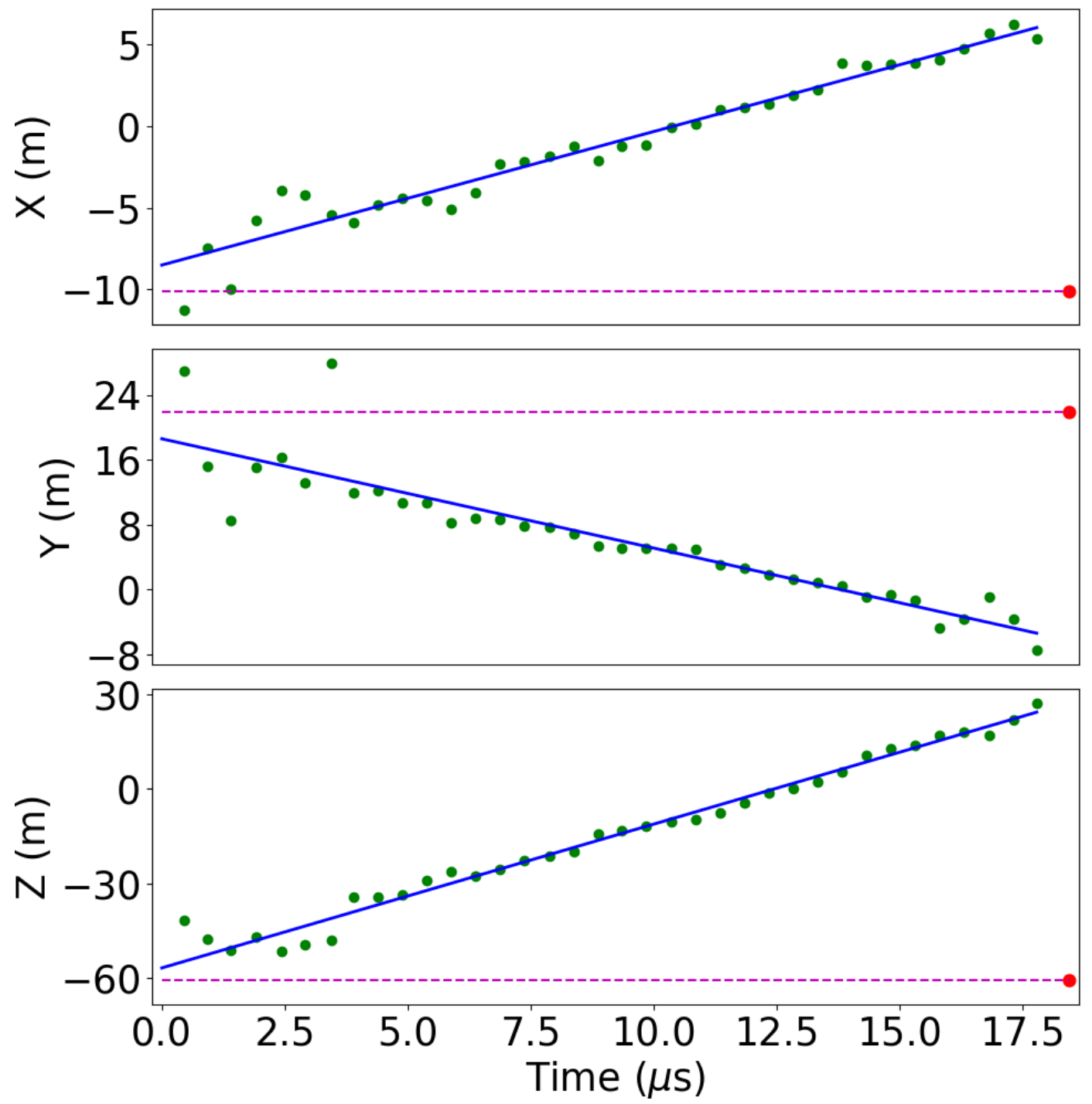

Figure 3. The above plots show a linear fit to the VHF source position versus time along the North (top), East (middle), and altitude (bottom) axes for the 2018 flash. The blue lines show linear fits to the data and the green dots indicate the location of the brightest pixel in each image. The scale on the left shows the distance from the imaging center in (Figure 1). The red dot and horizontal dashed line indicates location of the impulsive source shown in panel E of (Figure $1)$. 

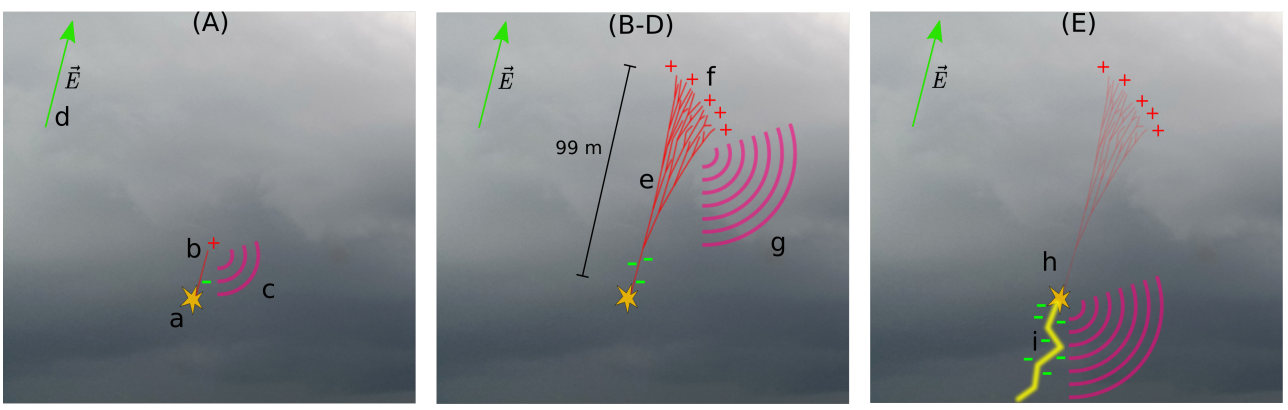

Figure 4. Sketch of proposed initiation process based on observations. The labels A-E indicate the corresponding panels in (Figure 1). Note that while we highlight that streamers are causing the motion of positive charge upward, this is truly due to electrons moving downward as ions are massive and do not move much by comparison.

first VHF radiation is produced (c) as a result of the streamer formation. The direction of the ambient field is indicated by the green arrow (d). The middle panel shows the development of the streamer avalanche (e) and fast upward propagation as a result of the initial streamer growing and splitting multiple times. Note that the widening of the avalanche is inferred from increase in image intensity, since work is still needed to clarify how the intensity profile of imaged pulses in (Figure 1) relate to physical source shape. The avalanche growth results in significant movement of positive charge mostly in an upward direction (f) and the production of a much larger VHF signal (g). The last panel on the right shows formation of the hot negative leader channel near the start of avalanche (h) due to the accumulation of excess negative charge at the tip. Also shown is the impulsive imager located sources from the formation of the first lightning leader (i) (Petersen et al., 2008).

Based on recent results of radio observations, it has been reported that some lightning flashes begin with what are known as a narrow bipolar events (NBEs) (Rison et al., 2016). NBEs are highly energetic bipolar waveforms that are detectable in VHF. They are believed to be the result of the process of fast breakdown of virgin air or an avalanche of streamers that precondition the initiation region to enable lightning initiation (Rison et al., 2016; Tilles et al., 2019; Liu et al., 2019). NBEs typically have an e-folding length between 9 and $32 \mathrm{~m}$ and are expected to be the result of particularly powerful discharges that are not observed with every lightning flash. Beamforming produces images of initiation events with much higher sensitivity and precision than typically reported NBEs (Rison et al., 2016). The observations reported in this work share a compatible e-folding length, but have an order of magnitude slower propagation speed, more compact avalanche region, and are much less powerful than reported NBEs (Rison et al., 2016; Tilles et al., 2019). As a result, what we see is likely the more common form of lightning initiation, of which we image in unprecedented detail. We show this via a true three-dimensional representation of the streamers, their collective trajectory, and the increase in power as they propagate during the initiation event.

A study by Lyu et al (2019) suggested that there were two distinct mechanisms for initiation, one that results in NBEs and another characterized by sub-microsecond VHF pulses with no identifiable fast breakdown signature (Lyu et al., 2019). With LOFAR, we show that these two mechanisms have compatible e-folding lengths, which indicates that the underlying electric fields may be similar in magnitude. What differs in our observations is the propagation speed and smaller total length of discharge. This suggests that the total high field region is shorter, however this also implies that the underlying mechanisms are the same. A smaller high field region would result in less of a development of the streamer avalanche and no NBE. For individual streamers, it is known that 
they exponentially accelerate and expand outward as they propagate, in addition to exponentially increasing in radiative power (Luque \& Ebert, 2014; Attanasio et al., 2019). Our observations show that for a system of streamers the properties are entirely different, and cannot be explained by a simple superposition of individual streamers. This poses a significant challenges to models, as the velocity of the front of the discharge of many individual streamers remains constant with radiation increasing exponentially. There must be a process which maintains this velocity and growth which has yet to be explained.

Our data supports the idea that cascading streamers initiate lightning when conditions are optimal (?, ?). Streamers can increase in number and produce VHF in the initiation region, as indicated by the ramp-up in intensity from background to near the rate of impulsive imager located sources. The first impulsive sources are observed to initiate at the location of the hypothesized streamer inception point. Interferometric beamforming locates these sources, showing this motion on meter scale and the overall increase in power of the streamer avalanche as it forms. This process provides a detailed 3D representation of the trajectory and reports an e-folding length that is consistent with previously published observations of fast breakdown in narrow bipolar events (NBEs) (Rison et al., 2016). Further studies will determine if this is the unique cause of lightning initiation, however the results we report here based on observations of lightning with LOFAR in VHF show significant advancement to the understanding of the physical processes of initiation through successfully imaging the initial stages of the formation of lightning.

\section{Acknowledgments}

\section{Author Contributions}

C.S. drafted the manuscript and completed data analysis. J.D., N.L., O.S., and B.M.H. contributed to critical review of main text and interpretation of results. O.S and B.M.H. developed the interferometry software for this study. S.B. and A.N. provided feedback and review. S.t.V. performed data calibration and acquisition.

\section{Data Availability}

Figures in this work were created with the Matplotlib Python package (Caswell et al., 2019). Data are located on the LOFAR Long Term Archive and can be downloaded after setting up a LOFAR LTA account and through following the instructions for "Staging Transient Buffer Board Data" (ASTRON, n.d.) using the wget software package as follows: wget -no-check-certificate https:// lofar-download.grid.surfsara.nl/lofigrid/SRMFifoGet.py?surl=srm:// srm.grid.sara.nl/pnfs/grid.sara.nl/data/lofar/ops/TBB/lightning/ L664182\_D20180809T141413.250Z\_"station"\_R000\_tbb.h5 and "station" is replaced with one of the names of the LOFAR stations: CS001, CS002, CS003, CS004, CS005, CS006, CS007, CS011, CS013, CS017, CS021, CS024, CS026, CS028, CS030, CS031, CS032, CS101, CS103, RS106, CS201, RS205, RS208, RS210, CS301, CS302, RS305, RS306, RS307, RS310, CS401, RS406, RS407, RS409, CS501, RS503, RS508, or RS509.

\section{Funding}

This research was supported in part through the University of New Hampshire AFOSR Grants No. FA9550-16-1-0396 and No. FA9550-18-1-0358.

\section{References}

ASTRON, L. (n.d.). LOFAR Long Term Archive Access. 
https://www.astron.nl/lofarwiki/doku.php?id=public:lta_howto.

Attanasio, A., Krehbiel, P. R., \& da Silva, C. L. ～(2019). Griffiths and Phelps Lightning Initiation Model, Revisited. Journal of Geophysical Research: Atmospheres, 124(14), 8076-8094. doi: 10.1029/2019JD030399

Caswell, T. A., Droettboom, M., Lee, A., Hunter, J., Firing, E., Stansby, D., ... Ivanov, P. (2019, December). Matplotlib/matplotlib v3.1.2. Zenodo. doi: 10.5281/zenodo.3563226

da Silva, C. L., \& Pasko, V. P. (2013). Dynamics of streamer-to-leader transition at reduced air densities and its implications for propagation of lightning leaders and gigantic jets. Journal of Geophysical Research: Atmospheres, 118(24), 13,561-13,590. doi: 10.1002/2013JD020618

Dubinova, A., Rutjes, C., Ebert, U., Buitink, S., Scholten, O., \& Trinh, G. T. N. (2015, June). Prediction of Lightning Inception by Large Ice Particles and Extensive Air Showers. Physical Review Letters, 115(1), 015002. doi: 10.1103/PhysRevLett.115.015002

Dwyer, J. R., \& Uman, M. A. (2014, January). The physics of lightning. Physics Reports, 534 (4), 147-241. doi: 10.1016/j.physrep.2013.09.004

Griffiths, R. F., \& Phelps, C. T. (1976). A model for lightning initiation arising from positive corona streamer development. Journal of Geophysical Research (18961977), 81 (21), 3671-3676. doi: 10.1029/JC081i021p03671

Hare, B. M., Scholten, O., Bonardi, A., Buitink, S., Corstanje, A., Ebert, U., ... Winchen, T. (2018). LOFAR Lightning Imaging: Mapping Lightning With Nanosecond Precision. Journal of Geophysical Research: Atmospheres, 123(5), 2861-2876. doi: 10.1002/2017JD028132

(KNMI). (2018, August). Radar precipitation above the Netherlands. http://geoservices.knmi.nl/viewer2.0.

Liu, N., Dwyer, J. R., Tilles, J. N., Stanley, M. A., Krehbiel, P. R., Rison, W., ... Wilson, J. G. (2019). Understanding the Radio Spectrum of Thunderstorm Narrow Bipolar Events. Journal of Geophysical Research: Atmospheres, 124(17-18), 10134-10153. doi: 10.1029/2019JD030439

Liu, N., Kosar, B., Sadighi, S., Dwyer, J. R., \& Rassoul, H. K. (2012, July). Formation of Streamer Discharges from an Isolated Ionization Column at Subbreakdown Conditions. Physical Review Letters, 109(2), 025002. doi: 10.1103/PhysRevLett.109.025002

Luque, A., \& Ebert, U. (2014, January). Growing discharge trees with self-consistent charge transport: The collective dynamics of streamers.

New Journal of Physics, 16(1), 013039. doi: 10.1088/1367-2630/16/1/013039

Lyu, F., Cummer, S. A., Qin, Z., \& Chen, M. (2019). Lightning Initiation Processes Imaged With Very High Frequency Broadband Interferometry. Journal of Geophysical Research: Atmospheres, 124(6), 2994-3004. doi: 10.1029/2018JD029817

Marshall, T., Bandara, S., Karunarathne, N., Karunarathne, S., Kolmasova, I., Siedlecki, R., \& Stolzenburg, M. (2019, March). A study of lightning flash initiation prior to the first initial breakdown pulse. Atmospheric Research, 217, 10-23. doi: 10.1016/j.atmosres.2018.10.013

Marshall, T., Stolzenburg, M., Karunarathna, N., \& Karunarathne, S. (2014). Electromagnetic activity before initial breakdown pulses of lightning. Journal of Geophysical Research: Atmospheres, 119(22), 12,558-12,574. doi: 10.1002/2014JD022155

Petersen, D., Bailey, M., Beasley, W. H., \& Hallett, J. (2008). A brief review of the problem of lightning initiation and a hypothesis of initial lightning leader formation. Journal of Geophysical Research: Atmospheres, 113(D17). doi: 10.1029/2007JD009036

Phelps, C. T. (1974, January). Positive streamer system intensification and its possible role in lightning initiation. Journal of Atmospheric and Terrestrial Physics, 
36 (1), 103-111. doi: 10.1016/0021-9169(74)90070-1

Rison, W., Krehbiel, P. R., Stock, M. G., Edens, H. E., Shao, X.-M., Thomas, R. J., ... Zhang, Y. (2016, February). Observations of narrow bipolar events reveal how lightning is initiated in thunderstorms. Nature Communications, 7(1), 10721. doi: $10.1038 /$ ncomms10721

Scholten, O., Hare, B., Dwyer, J., Liu, N., Sterpka, C., Buitink, S., ... ter Veen, S. (2021, April). Time resolved 3Dinterferometric imaging of a section of a negative leader with LOFAR. arXiv:2104.11908 [astro-ph, physics:physics].

Scholten, O., Hare, B. M., Dwyer, J., Sterpka, C., Kolmašová, I., Santolík, O., ... Winchen, T. (2021). The Initial Stage of Cloud Lightning Imaged in High-Resolution. Journal of Geophysical Research: Atmospheres, 126(4), e2020JD033126. doi: 10.1029/2020JD033126

Shi, F., Liu, N., Dwyer, J. R., \& Ihaddadene, K. M. A. (2019). VHF and UHF Electromagnetic Radiation Produced by Streamers in Lightning. Geophysical Research Letters, 46 (1), 443-451. doi: 10.1029/2018GL080309

Shi, F., Liu, N., \& Rassoul, H. K. (2016). Properties of relatively long streamers initiated from an isolated hydrometeor. Journal of Geophysical Research: Atmospheres, 121(12), 7284-7295. doi: 10.1002/2015JD024580

Stock, M. G., Akita, M., Krehbiel, P. R., Rison, W., Edens, H. E., Kawasaki, Z., \& Stanley, M. A. (2014). Continuous broadband digital interferometry of lightning using a generalized cross-correlation algorithm. Journal of Geophysical Research: Atmospheres, 119(6), 3134-3165. doi: 10.1002/2013JD020217

Stolzenburg, M., Marshall, T. C., \& Karunarathne, S. (2020). On the Transition From Initial Leader to Stepped Leader in Negative Cloud-to-Ground Lightning. Journal of Geophysical Research: Atmospheres, 125(4), e2019JD031765. doi: 10.1029/2019JD031765

Taylor, G. B., Carilli, C. L., \& Perley, R. A. (1999). Synthesis Imaging in Radio Astronomy II (Vol. 180). Astronomical Society of the Pacific.

Tilles, J. N., Liu, N., Stanley, M. A., Krehbiel, P. R., Rison, W., Stock, M. G., ... Wilson, J. (2019, April). Fast negative breakdown in thunderstorms. Nature Communications, 10(1), 1-12. doi: 10.1038/s41467-019-09621-z 